\title{
EFEITOS DAS DIFERENTES FREQUÊNCIAS DA ELETROESTIMULAÇÃO NEUROMUSCULAR SOBRE A FADIGA MUSCULAR: ENSAIO CLÍNICO CRUZADO RANDOMIZADO
}

\author{
Júlio César A. P. Malafaia ${ }^{1 *}$, Rafael Nunes Silva ${ }^{1}$ E Felipe Sampaio Jorge ${ }^{2}$
}

MALAFAIA, J.C.A.P; SILVA, R.N. \& JORGE,F.S. Efeitos das diferentes frequências da eletroestimulação neuromuscular sobre a fadiga muscular: ensaio clínico cruzado randomizado. Perspectivas Online: Biológicas \& Saúde. v. 8, n 28, p.1-14, 2018.

\section{RESUMO}

Introdução: Existem poucas descrições na literatura que comprovem os efeitos da aplicação de diferentes frequências sobre $o$ organismo humano. Este estudo quer possibilitar a investigação sobre os efeitos da corrente alternada através da avaliação do período da fadiga muscular, potência, força muscular e alterações do metabolismo da glicose. Metodologia: Foi realizado um ensaio clínico cruzado randomizado, com dez voluntários sadios, sexo masculino, sedentários, idade entre 18 e 35 anos. Foram avaliados os dados antropométricos: massa, estatura, perimetria do braço relaxado e contraído. No experimento foi realizado à randomização das frequências e os tipos de dispositivos utilizados na EENM. Foi coletado uma pequena amostra de sangue, antes de iniciar e após intervenção. Foi coletado o sinal EMG e foi usada uma célula de carga para obtenção da força máxima gerada na contração. Resultados: Após o teste de CVM não se verificou diferença do pico de força entre as frequências da EENM. Não houve diferença na atividade muscular em todas as frequências aplicadas. Não demonstrou diferença nos valores de RMS, e na Frequência Mediana (Fmd) não foi detectado diferença nos valores após o experimento. Na corrente AUSSIE, verificouse que a glicemia pós-teste diminuiu nas frequências de $60 \mathrm{~Hz}$ e $120 \mathrm{~Hz}$ e na corrente alternada que a glicemia diminuiu nas frequências de $90 \mathrm{~Hz}$ e $120 \mathrm{~Hz}$. Conclusão: Pode-se assegurar que as diferentes frequências da EENM não foram capazes de gerar alterações na produção de força e nem na geração de fadiga muscular pós EENM. Porém verificou-se que as frequências possuem um potencial no consumo de glicose.

Palavras-chave: Fisioterapia, Eletroestimulação Neuromuscular, Eletroterapia, TENS. 


\begin{abstract}
Few descriptions in the literature prove the effects of the application of different frequencies on the human organism. This study intends to enable the investigation of the effects of alternating current through the evaluation of the period of muscle fatigue, power, muscular strength and alterations of glucose metabolism. A randomized crossover clinical trial was carried out with ten healthy volunteers, male, sedentary, age between 18 and 35 years. The anthropometric data were evaluated: mass, height, perimetry of the relaxed and contracted arm. The experiment was carried out to randomize the frequencies and types of devices used in the NMES. A small blood sample was collected before and after intervention. The EMG signal was collected and a load cell was used to obtain the maximum force generated in the

contraction. After the CVM there was no difference in the peak of force between the NMES frequencies. There was no difference in muscle activity in all applied frequencies. There was no difference in the RMS values, and in the Median Frequency (Fmd) no difference was detected in the values after the experiment. In the AUSSIE current, it was verified that the post-test glucose decreased in the frequencies of $60 \mathrm{~Hz}$ and $120 \mathrm{~Hz}$ and in the alternating current that the blood glucose after test decreased in the frequencies of 90 $\mathrm{Hz}$ and $120 \mathrm{~Hz}$. It can be ensured that the different frequencies of NMES were not able to generate changes in strength production nor in the generation of muscle fatigue after NMES. However, it was verified that the frequencies have a potential in glucose consumption.
\end{abstract}

Key-words: Physiotherapy, Neuromuscular Electrostimulation, Electrotherapy, TENS.

\footnotetext{
${ }^{1}$ Institutos Superiores de Ensino do CENSA -ISECENSA - Aluno Bolsista de Iniciação Científica PIBIC - Rua Salvador Correa, 139, Centro, Campos dos Goytacazes, RJ, CEP: 28035-310, Brasil.

${ }^{2}$ Aluno Voluntário de Iniciação Científica PIBIC/ISECENSA

${ }^{3}$ Pesquisador do Núcleo de Pesquisa em Fisioterapia Traumato Ortopédica Funcional - NUPEFITO/ISECENSA

(*)e-mail: juliomalafaia96@gmail.com

Data de recebimento: 28/07/2018 Aceito para publicação: 13/12/2018
}

Persp. online: biol. \& saúde, Campos dos Goytacazes, 28 (8), 1-14, 2018

seer.perspectivasonline.com.br 


\section{INTRODUÇÃO}

Atualmente existem poucas descrições, na literatura e em trabalhos científicos, que comprovem os efeitos da aplicação de diferentes frequências sobre o organismo humano. Este estudo quer possibilitar uma investigação sobre os efeitos da corrente alternada sobre o organismo humano, através da avaliação do período da fadiga muscular, potência, força muscular e alterações do metabolismo da glicose.

O exercício físico promove a biogênese mitocondrial, aumenta a capacidade de produção de ATP e neutralização de espécies reativas de oxigênio/reactive oxygen species (ROS). Também aumenta o crescimento e sinalização da síntese de proteínas, reduz a apoptose celular e as vias de degradação proteica, efeitos que previnem a atrofia muscular esquelética. Um estudo analisou o fator de transcrição mitocondrial A (FTMA) que protege o DNA mitocondrial da degradação via ROS e desencadeia a ativação de fatores de transcrição mitocondrial, prevenindo a disfunção mitocondrial e redução a atrofia muscular esquelética. A atividade física através da FTMA pode ser o tratamento contra os efeitos negativos associados ao desuso dos músculos, no caso de pacientes em repouso ou imobilização após uma cirurgia (THEILEN; KUNKEL; TYAGI, 2017).

A eletroestimulação muscular é definida como um método de condução da energia elétrica, que estimula a contração muscular, sem a necessidade de geração de um pulso elétrico do sistema nervoso pelo indivíduo. É um mecanismo que utiliza uma corrente alternada de baixa intensidade para estimular uma contração muscular fisiológica, a analgesia e a correção das atividades metabólicas dos sistemas endócrino, vascular e tegumentar. Os objetivos de eletroestimulação tornam-se mais abrangentes quando compreendemos as bases da corrente elétrica e a sua interação com os tecidos biológicos, já que eles são relacionados com a função de cada tecido e na forma que eles podem ser estimulados (AGNE, 2015).

A EENM pode ser uma alternativa, que promova a contração muscular, a prática de exercícios físicos durante períodos de desuso muscular por causa de doença ou lesão (DIRKS et al., 2014). Um estudo avaliou, através de biópsia muscular, que apenas uma sessão de EENM é necessária para incrementar as taxas de síntese proteicas do músculo esquelético na recuperação aguda de diabéticos tipo 2. A EENM pode ser aplicada de forma efetiva, em indivíduos idosos, para reverter o quadro de perda de massa muscular em períodos de repouso e imobilização (WALL et al., 2012).

Considerada uma técnica de treinamento muscular adequada e eficaz para melhorar ou até mesmo reduzir o declínio da força física em idosos, a EENM é utilizada na melhora do nível funcional desses indivíduos. Um estudo de revisão sistemática identificou que um programa de treinamento de quatro semanas, com periodicidade de três vezes por semana e utilizando uma frequência de pulsos entre 25 e $50 \mathrm{~Hz}$, pode promover efeitos positivos na melhora da marcha e equilíbrio dos idosos. Concluíram que a EENM pode ser usada como uma técnica segura e eficaz e deve ser utilizada associada ou não a atividade física voluntária tradicional, o que reduzirá os riscos de queda e promoverá qualidade de vida aos idosos (LANGEARD et al., 2017).

Com isso o presente estudo avaliou qual a influência das diferentes frequências da EENM em dois eletroestimuladores (AUSSIE e corrente alternada) na produção de força induzida eletricamente e na fadiga muscular após EENM. Além de avaliar os diferentes efeitos das frequências no consumo de glicose durante a EENM.

Persp. online: biol. \& saúde, Campos dos Goytacazes, 28 (8), 1-14, 2018

seer.perspectivasonline.com.br 


\section{METODOLOGIA}

Foi realizado um ensaio clínico cruzado randomizado com 10 voluntários do sexo masculino, sedentários (sem realizar atividade física no mínimo por três meses) e com idade entre 18 e 35 anos. Os critérios de exclusão foram indivíduos que praticavam atividade física regular, com idade menor que 18 anos e maior que 35 anos, indivíduos que sofreram lesões graves osteomioarticulares no membro superior e que eram do sexo feminino.

Com o objetivo de organizar as ações, que foram executadas em todos os processos da intervenção, segue abaixo um organograma dividindo todas as etapas descritas na metodologia (Figura 1).

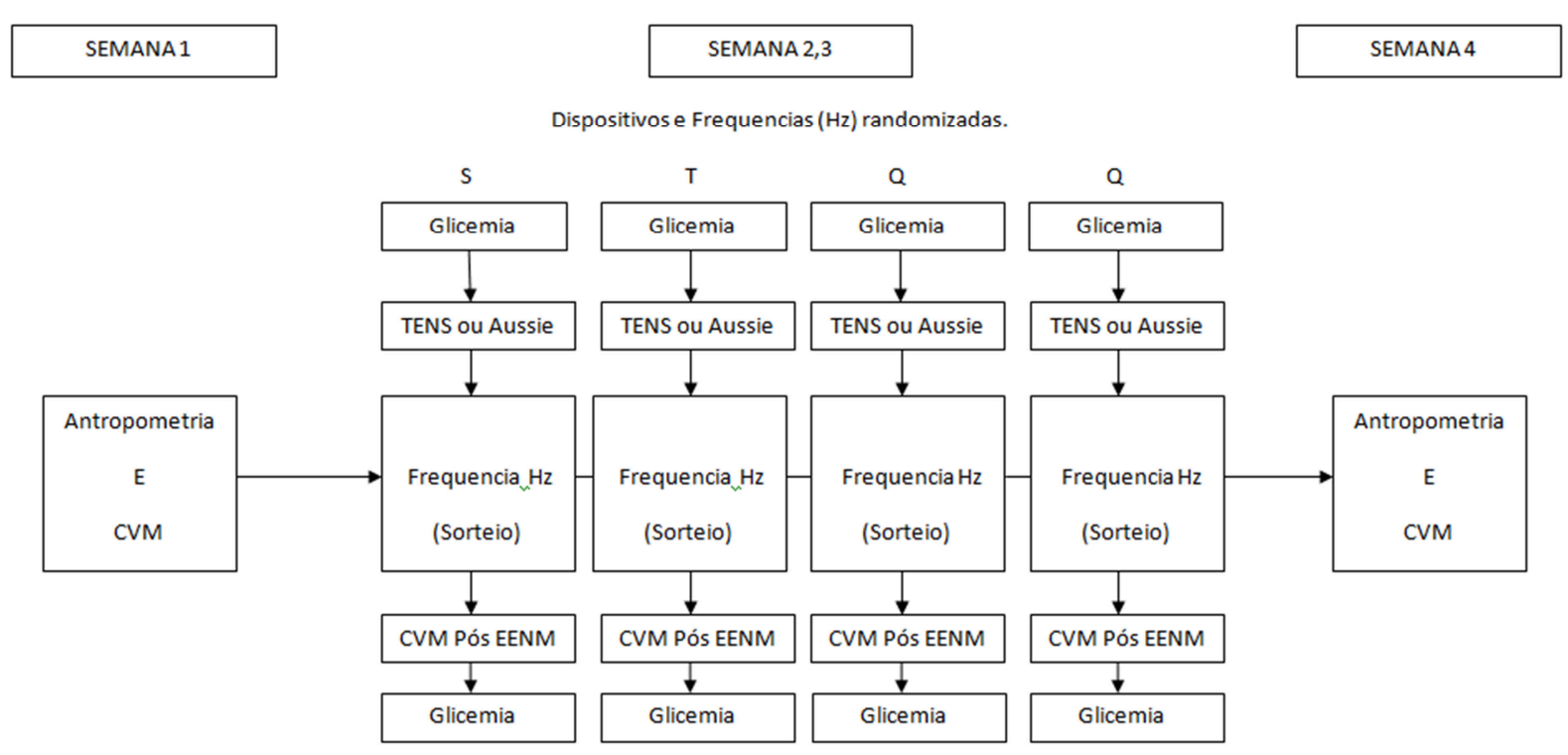

Figura 1. Organograma dividindo em etapas as atividades metodológicas.

\section{Avaliação Basal}

Com o objetivo de se verificar a homogeneidade da amostra, foram avaliados os seguintes dados antropométricos: massa, estatura, perimetria do braço com flexão de cotovelo a noventa graus relaxado e contraído do membro dominante. Para garantir que o membro estivesse a noventa graus, foi utilizado um Goniômetro.

Foi realizado um teste de Contração Voluntária Máxima (CVM) isométrica do músculo Bíceps Braquial, utilizou-se um eletromiógrafo (com oito canais, EMG System Brasil com $2000 \mathrm{~Hz}$ de taxa de amostragem) com o objetivo de capturar os sinais elétricos gerados na fibra muscular. Os eletrodos foram posicionados segundo o protocolo SENIAM (Disponível em: http://www.seniam.org). O paciente estava com o tronco e os braços apoiados sobre um suporte no formato triangular, com o cotovelo a noventa graus de flexão, segurando uma barra ligada a um dinamômetro (célula de carga com saturação em $200 \mathrm{KgF}$ ) com o membro dominante (Figura 2). 


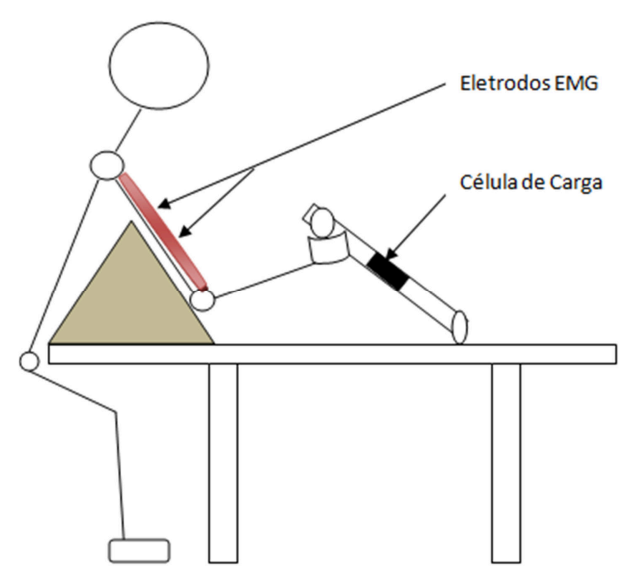

Figura 2. Esquema do protocolo de posicionamento do estudo. Com esse protocolo é possível verificar a posição de realização do teste CVM.

No teste de CVM, foi gerado um gráfico que representa o comportamento da força muscular durante os 180 segundos. O Eixo " $y$ " representa a força gerada $(\mathrm{KgF})$ e o eixo "x" é o tempo (segundos). O valor mais alto de " $y$ ", no gráfico, foi considerado como pico de força. O ponto que equivale a $50 \%$ do valor do pico de força, foi localizado no eixo "x", e indicou o tempo de ocorrência da fadiga muscular. O período formado entre o tempo de geração do pico de força e o tempo de fadiga muscular mostrou uma área gráfica que representa area under curve - AUC (Figura 3).

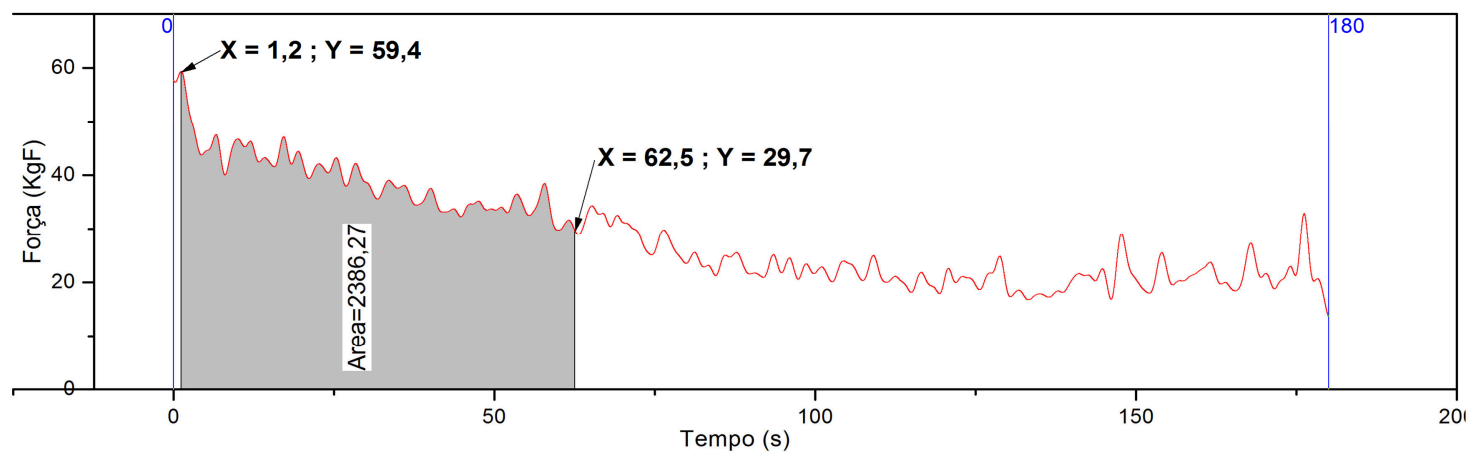

Figura 3. Força gerada no teste de CVM durante 180 segundos com a indicação do pico de força (KgF), tempo de fadiga (segundos) e a AUC.

\section{Experimento}

Os participantes do experimento foram orientados a fazer jejum de quatro horas antes de cada intervenção. Foi coletado uma pequena amostra de sangue, puncionada na Polpa Digital do Quarto Quirodáctilo, antes de iniciar e depois de cada experimento, com objetivo de avaliar o comportamento glicêmico pré e pós intervenção. Utilizou-se um medidor de glicose sanguínea e tiras de glicose da marca On Call Plus. 
Os dispositivos foram: corrente Australiana com a forma de onda Senoidal, Bifásica e simétrica programada no modo contínuo, com frequência portadora de $1,0 \mathrm{KHz}$ e tempo de burst 2 ms. Outro dispositivo utilizado foi a corrente alternada com forma de onda Retangular, Bifásica e simétrica, $500 \mathrm{~ms}$ de tempo de pulso e com o tempo de aplicação da corrente e posicionamento descritos acima. Foi utilizada uma célula de carga para aquisição da força gerada pelo estímulo elétrico. Para a realização da EENM, os parâmetros da frequência e os dispositivos foram randomizados, sendo as frequências avaliadas: $30 \mathrm{~Hz} ; 60 \mathrm{~Hz} ; 90 \mathrm{~Hz} ; 120 \mathrm{~Hz}$, com washout de vinte e quatro horas entre a aplicação delas.

Com o objetivo de se avaliar o torque máximo gerado por cada frequência foi utilizado um tempo de aplicação da corrente de cinco minutos (300 segundos) na intensidade máxima tolerada (mA). O músculo Bíceps Braquial estava em contração máxima induzida eletricamente (CMIE), fixo a uma angulação de noventa graus de flexão de cotovelo (Figura 4).

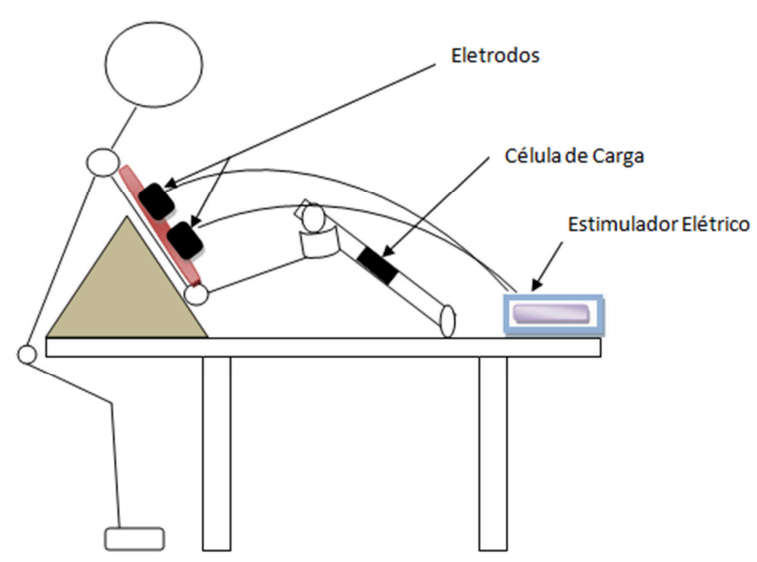

Figura 4. Esquema do protocolo de posicionamento do estudo. Com esse protocolo é possível avaliar a força gerada pela EENM.

Após cada EENM, foi iniciado um novo teste de CVM isométrica, do músculo Bíceps Braquial, durante três minutos (180 segundos), a fim de se avaliar o efeito de depleção de força pós EENM.

\section{Avaliação Final}

Objetivando-se verificar o comportamento do músculo após a realização de todos os testes previstos, foi realizada uma avaliação da CVM do indivíduo, durante três minutos. Este teste foi idêntico ao realizado na avaliação basal e teve como principal objetivo avaliar possíveis adaptações teciduais aos estímulos elétricos.

\section{Análise de dados}

Foi utilizado um software de análise de dados, Origin Pro 2016, para tratamento estatístico. Os gráficos brutos gerados pelo Dinamômetro de Força foram suavizados a 1500 pontos por janela

Persp. online: biol. \& saúde, Campos dos Goytacazes, 28 (8), 1-14, 2018

seer.perspectivasonline.com.br 
utilizando o filtro FFT, para retirar os possíveis artefatos do gráfico. Após suavização do gráfico foi possível localizar com precisão, o Pico de Força gerado e tempo de fadiga. Com a realização do cálculo da Integral foi possível obter a AUC, numa janela de tempo específica (do Pico de Força até o Tempo de Fadiga), que corresponde à realização de trabalho muscular.

No sinal EMG foi realizado o Root Mean Square/Raiz Quadrada da Média Quadrática (RMS) a 500 pontos por janela, onde era possível visualizar todo gráfico com valores positivos. Os dados de CVM Pós EENM durante 180 segundos obtinham o sinal eletromiográfico, que foram divididos em quatro quadrantes por tempo, de 0 a $45 \mathrm{~s}, 45$ a 90 s, 90 a 135 s e 135 a $180 \mathrm{~s}$, para obter o valor RMS e Fmd durante todo o protocolo avaliando-se assim o comportamento muscular do indivíduo.

Para o conhecimento do comportamento da amostra realizou-se o teste de normalidade de Shapiro-wilk, verificando-se que todos os dados tinham comportamento normal $(\mathrm{P}>0,05)$. Com isso adotou-se o teste Two Way ANOVA com posto hoc de Tukey, com índice de significância de 5\%. Para avaliação do tamanho do efeito, nas variáveis com diferença significativa, adotou-se a diferença entre médias com intervalo de confiança de $95 \%$.

\section{RESULTADOS}

A caracterização da amostra foi feita através da idade, estatura, massa corporal e IMC da amostra. Os dados foram expressos na forma de média, desvio padrão e o coeficiente de variação percentual (CV\%) foi calculado a fim de se confirmar a homogeneidade da amostra (Tabela 1).

Tabela 1 - Dados antropométricos da amostra. Dados expressos na forma de média \pm desvio padrão. Verifica-se CV\% foi menor que $30 \%$, o que demonstrou uma amostra homogênea.

\begin{tabular}{ccc}
\hline Idade (anos) & $23,80 \pm 3,85$ & 16,18 \\
Estatura $(\mathrm{m})$ & $1,77 \pm 0,07$ & 3,95 \\
Massa $(\mathrm{Kg})$ & $78,59 \pm 11,48$ & 14,61 \\
$\mathrm{IMC}\left(\mathrm{m} / \mathrm{Kg}^{2}\right)$ & $25,17 \pm 4,23$ & 16,81 \\
\hline
\end{tabular}

Ao se analisar o comportamento das diferentes frequências de EENM, verificou-se um comportamento similar entre elas $(p>0,05)$ na geração de força durante o teste de 300 segundos, em CMIE. A geração de força foi de aproximadamente $20,0 \mathrm{KgF}$ nas duas correntes estudadas e equivale, aproximadamente, a terça parte do pico de força média ( $60 \mathrm{KgF})$ gerada no teste de CVM basal (Figura 5). 


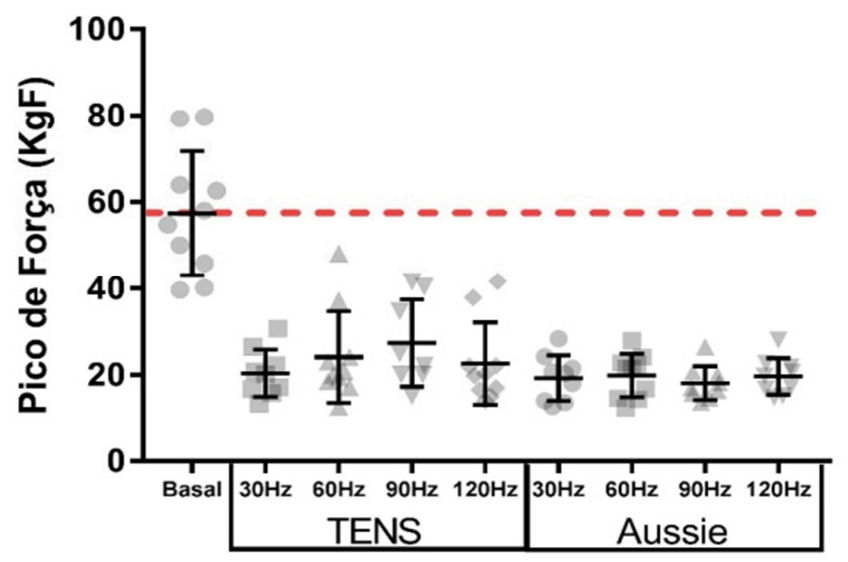

Figura 5. Valores de pico de força gerados por cada frequência. Não se verificou diferença entre o pico de força nas frequências de EENM ( $p>0,05)$.

$\mathrm{Na}$ avaliação de força obtida após a EENM, não se verificou diminuição do pico de força $(\mathrm{p}>0,05)$ nas diferentes frequências analisadas durante o teste de 180 segundos. Dessa forma, não se identificou fadiga muscular induzida pela EENM (Figura 6).

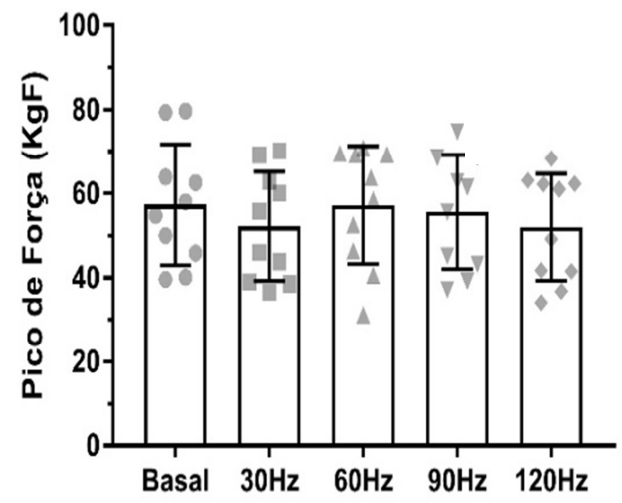

B

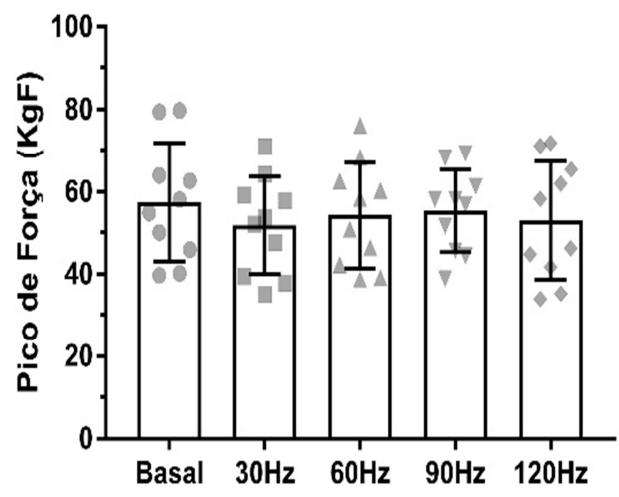

Figura 6. (A) Valores do pico de força na CVM após a corrente AUSSIE. (B) Valores do pico de força na CVM após a corrente TENS. Não se verificou diferença entre as frequências testadas

$$
(\mathrm{p}>0,05) \text {. }
$$

Através da análise da AUC foi possível determinar a atividade muscular durante os 180 segundos, no teste de CVM após o experimento com as frequências. Não houve diferença na atividade muscular em todas as frequências aplicadas (Figura 7). 
A

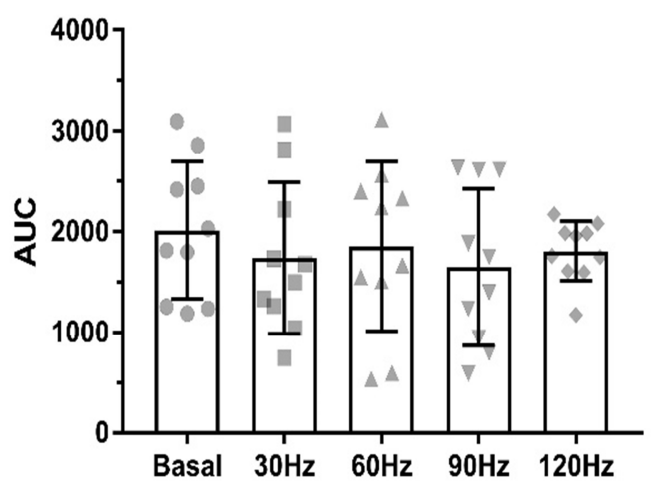

B

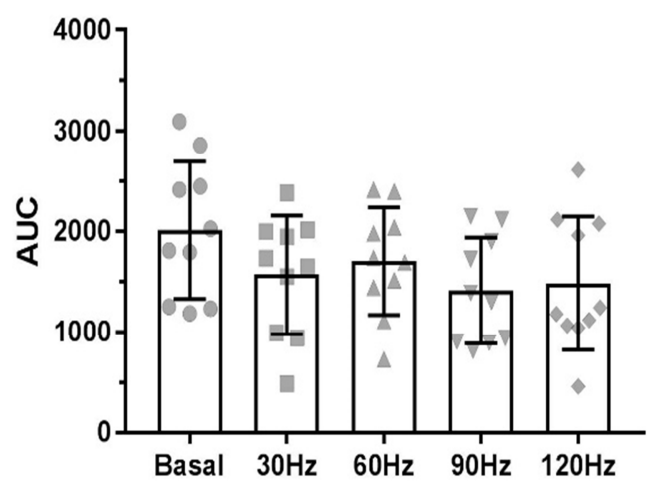

Figura 7. (A) Área sob a curva na CVM após a corrente TENS. (B) Área sob a curva na CVM após a corrente AUSSIE. Não se verificou diferença entre as frequências testadas ( $p>0,05)$.

Para avaliar o comportamento EMG dos músculos após EENM foram gerados valores de RMS que identificam o recrutamento das fibras musculares. Não se verificou diferença nos valores de RMS após a aplicação de cada frequência analisada ( $p>0,05)$ (Tabela 2).

Tabela 2. Valores de RMS no teste CVM após a aplicação das frequências. Não se verificou diferença nas frequências testadas $(\mathrm{p}>0,05)$. Observação: Os dados de RMS estão dispostos na tabela na forma de média \pm desvio padrão.

RMS

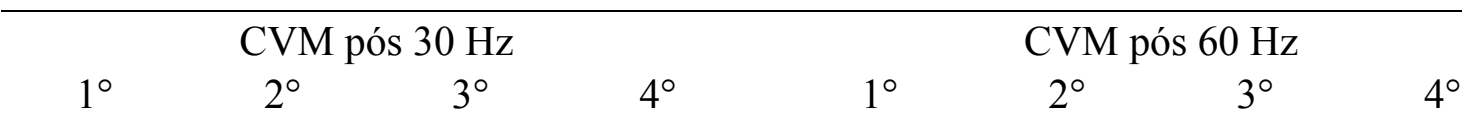

Quadrant Quadrant Quadrant Quadrant Quadrant Quadrant Quadrant Quadrant

\begin{tabular}{|c|c|c|c|c|c|c|c|c|}
\hline & e & e & e & e & e & e & e & $\mathrm{e}$ \\
\hline \multirow{2}{*}{ TENS } & 346,38 & 267,61 & 204,531 & 176,48 & 290,92 & 226,29 & 153,88 & 151,46 \\
\hline & $\pm 282,64$ & $\pm 207,86$ & $\pm 106,73$ & $\pm 94,10$ & $\pm 236,97$ & $\pm 235,70$ & $\pm 136,14$ & $\pm 131,01$ \\
\hline \multirow{3}{*}{$\begin{array}{c}\text { AUSSI } \\
\mathrm{E} \\
\end{array}$} & 268,63 & 190,11 & 157,82 & 163,69 & 256,74 & 185,91 & 157,50 & 150,85 \\
\hline & $\pm 203,38$ & $\pm 115,80$ & $\pm 88,25$ & $\pm 117,50$ & $\pm 163,91$ & $\pm 78,43$ & $\pm 66,78$ & $\pm 75,14$ \\
\hline & \multicolumn{4}{|c|}{ CVM pós $90 \mathrm{~Hz}$} & \multicolumn{4}{|c|}{ CVM pós $120 \mathrm{~Hz}$} \\
\hline \multirow{2}{*}{ TENS } & 278,94 & 237,24 & 181,50 & 140,89 & 254,84 & 204,35 & 178,75 & 159,50 \\
\hline & $\pm 191,17$ & $\pm 174,40$ & $\pm 122,99$ & $\pm 87,44$ & $\pm 166,26$ & $\pm 121,60$ & $\pm 101,12$ & $\pm 70,27$ \\
\hline AUSSI & 214,99 & 186,73 & 158,26 & 153,42 & 235,85 & 206,37 & 147,79 & 135,85 \\
\hline $\mathrm{E}$ & $\pm 136,23$ & $\pm 109,48$ & $\pm 81,55$ & $\pm 74,23$ & $\pm 148,56$ & $\pm 107,33$ & $\pm 64,73$ & $\pm 65,90$ \\
\hline
\end{tabular}


Complementando a avaliação dos sinais EMGs foram gerados valores da Frequência mediana (Fmd) que representa o sincronismo das fibras musculares. Também não se verificou diferença nos valores de Fmd após a aplicação de cada frequência analisada $(\mathrm{p}>0,05)$ (Tabela3).

Tabela 3. Valores da Fmd no teste CVM após a aplicação das frequências. Não se verificou diferença nas frequências testadas ( $p>0,05)$. Observação: Os dados da Fmd estão dispostos na tabela na forma de média \pm desvio padrão.

Frequência mediana

\begin{tabular}{|c|c|c|c|c|c|c|c|c|}
\hline & \multicolumn{4}{|c|}{ CVM pós $30 \mathrm{~Hz}$} & \multicolumn{4}{|c|}{ CVM pós $60 \mathrm{~Hz}$} \\
\hline & $\underset{\mathrm{e}}{1^{\circ}}$ & $\begin{array}{c}2^{\circ} \\
\text { Quadrante }\end{array}$ & $\begin{array}{c}3^{\circ} \\
\text { Quadrante }\end{array}$ & $\begin{array}{c}4^{\circ} \\
\text { Quadrante }\end{array}$ & $\begin{array}{c}1^{\circ} \\
\text { Quadrante }\end{array}$ & $\begin{array}{c}2^{\circ} \\
\text { Quadrante }\end{array}$ & $\begin{array}{c}3^{\circ} \\
\text { Quadrante }\end{array}$ & $\begin{array}{l}4^{\circ} \\
\text { Quadrante }\end{array}$ \\
\hline TENS & $\begin{array}{c}69,22 \\
\pm 13,15\end{array}$ & $\begin{array}{c}65,74 \\
\pm 14,33\end{array}$ & $\begin{array}{c}69,25 \\
\pm 15,91\end{array}$ & $\begin{array}{c}70,11 \\
\pm 14,83\end{array}$ & $67,30 \pm 6,20$ & $\begin{array}{c}65,08 \\
\pm 11,11\end{array}$ & $\begin{array}{c}67,06 \\
\pm 11,10\end{array}$ & $\begin{array}{c}67,31 \\
\pm 12,69\end{array}$ \\
\hline \multirow[t]{2}{*}{ AUSSIE } & $\begin{array}{c}67,74 \\
\pm 11,58\end{array}$ & $\begin{array}{c}67,11 \\
\pm 12,38\end{array}$ & $\begin{array}{c}66,24 \\
\pm 11,37\end{array}$ & $\begin{array}{c}68,10 \\
\pm 12,17\end{array}$ & $70,37 \pm 7,06$ & $66,90 \pm 9,43$ & $66,99 \pm 9,66$ & $\begin{array}{c}69,50 \\
\pm 10,69\end{array}$ \\
\hline & \multicolumn{4}{|c|}{ CVM pós $90 \mathrm{~Hz}$} & \multicolumn{4}{|c|}{ CVM pós $120 \mathrm{~Hz}$} \\
\hline TENS & $\begin{array}{l}70,09 \\
\pm 8,81\end{array}$ & $\begin{array}{c}66,61 \\
\pm 10,74\end{array}$ & $\begin{array}{c}69,62 \\
\pm 12,53\end{array}$ & $70,32 \pm 9,44$ & $\begin{array}{c}73,59 \\
\pm 10,97\end{array}$ & $\begin{array}{c}69,68 \\
\pm 12,92\end{array}$ & $\begin{array}{c}70,35 \\
\pm 13,10\end{array}$ & $\begin{array}{c}71,10 \\
\pm 11,84\end{array}$ \\
\hline AUSSIE & $\begin{array}{l}72,91 \\
\pm 9,49\end{array}$ & $\begin{array}{c}71,62 \\
\pm 12,12\end{array}$ & $\begin{array}{c}71,16 \\
\pm 11,67\end{array}$ & $\begin{array}{c}70,80 \\
\pm 11,87\end{array}$ & $\begin{array}{c}73,60 \\
\pm 11,25\end{array}$ & $\begin{array}{c}69,62 \\
\pm 13,17\end{array}$ & $\begin{array}{c}71,01 \\
\pm 11,62\end{array}$ & $\begin{array}{l}71,51 \\
\pm 1,44\end{array}$ \\
\hline
\end{tabular}

Os níveis de consumo da glicose foram verificados para se analisar o efeito hipoglicemiante no organismo indivíduo submetido à variação das frequências da EENM. No experimento que utilizou a corrente AUSSIE, verificou-se que a glicose sanguínea (pós-teste) diminuiu nas frequências de $60 \mathrm{~Hz}$ e $120 \mathrm{~Hz}(\mathrm{p}<0,05)$, em relação ao valor de glicose pré-teste, e que não houve alteração da glicose sanguínea nas demais frequências $(p>0,05)$. Avaliando o intervalo de confiança, constatou-se que as frequências $60 \mathrm{~Hz}$ e $120 \mathrm{~Hz}$ gerou uma alteração clínica nos indivíduos avaliados (Figura $8-A$ ).

No experimento que utilizou a corrente TENS, observou-se que a glicose sanguínea diminuiu nas frequências mais altas de $90 \mathrm{~Hz}$ e $120 \mathrm{~Hz}(\mathrm{p}<0,05)$, em relação ao valor de glicose pré-teste, e que não houve alteração da glicose sanguínea nas demais frequências $(p>0,05)$. A análise do intervalo de confiança mostrou que a frequência de $120 \mathrm{~Hz}$ obteve um melhor resultado clínico nos indivíduos estudados (Figura 8-B). 
A

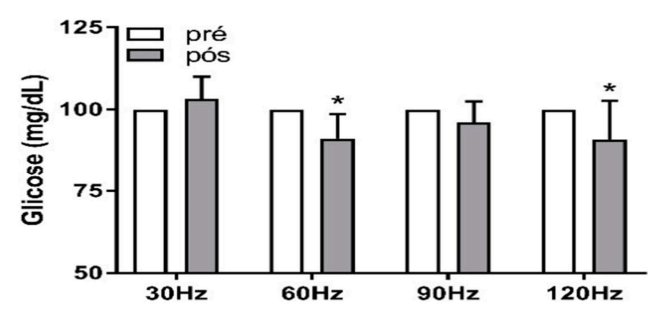

95\% Confidence Intervals (Sidak)

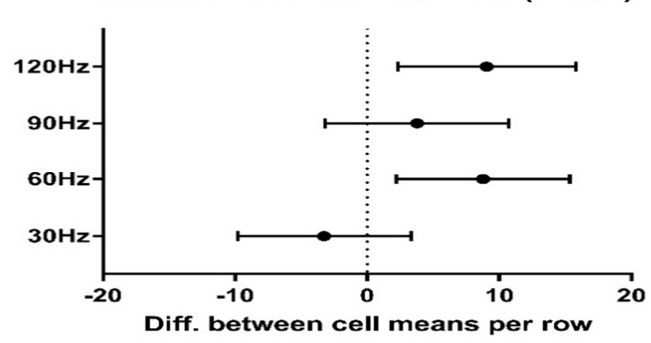

B

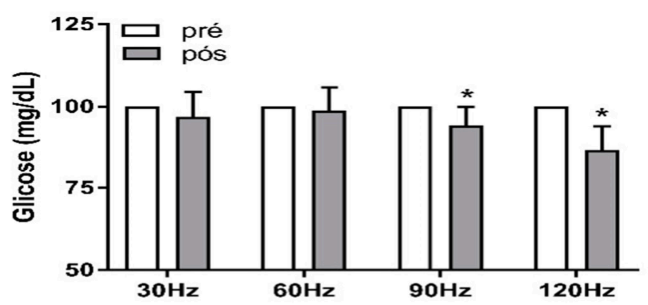

95\% Confidence Intervals (Bonferroni)

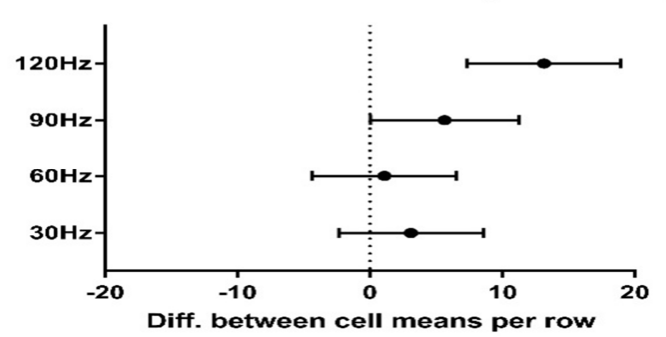

Figura 8. (A) Consumo da glicose sanguínea após cada experimento com a corrente AUSSIE. (B) Consumo da glicose sanguínea após cada experimento com a corrente TENS. Verificou-se diminuição da glicose sanguínea nas frequências $60 \mathrm{~Hz}$ e $120 \mathrm{~Hz}$ (AUSSIE) e $90 \mathrm{HZ}$ e $120 \mathrm{~Hz}$ (TENS), $(\mathrm{p}<0,05)$.

\section{DISCUSSÃO}

A variação das frequências não alterou o Pico de força, que corresponde ao torque máximo gerado pela EENM, o que mostra que o aumento da frequência não é fator que aumente o torque. Um estudo avaliou a Corrente Russa, Corrente Aussie, Corrente Pulsada com 500 ms e Corrente Pulsada com 200ms utilizando somente a frequência de 50Hz.Após realizar os testes revelaram que, não houveram diferenças no máximo de torque induzido eletricamente provocadas pela Corrente Aussie, Corrente Pulsada com 200ms e Corrente Pulsada com 500ms. A Corrente Russa gerou menos torque do que as demais. Concluíram que, Corrente Aussie ou Corrente Pulsada a 500ms e 200msforam igualmente eficazes em termos de produção de força. (WARD; OLIVER; BUCCELLA, 2006). Dados esses que corroboram com o presente estudo.

No atual estudo, independentemente da frequência utilizada, o torque gerado é igual em todas as frequências. A literatura aponta que de acordo com o aumento da frequência, maior será a força obtida eletricamente. O autor indica que para ocorrer contração prolongada e estável, deve-se utilizar frequências mais elevadas, de $50-70 \mathrm{~Hz}$ e pode variar entre $65-70 \mathrm{~Hz}$ para garantir a maior estabilidade da contração muscular. O presente estudo mostra que a menor frequência de $30 \mathrm{~Hz}$ até a maior de $120 \mathrm{~Hz}$, são iguais em gerar pico de força. O mesmo autor em uma literatura atualizada formulou programas de EENM, descritas como Programa hipertrofia ou força-resistência e programa de força explosiva, que consiste em utilizar frequências entre $60-80 \mathrm{~Hz}$ e $100-150 \mathrm{~Hz}$ para gerar maior torque respectivamente. (AGNE, 2004, 2015). Após EENM durante 300 segundos foi realizado um teste de CVM num período de 180 segundos com objetivo de avaliar o stress 
muscular. Os resultados demonstraram que a EENM não foi capaz de diminuir o desempenho, as frequências utilizadas não foram fator causal para gerar perda funcional (Figura 4A, B).

AUC foi usado para avaliar o trabalho muscular e é uma forma de analisar o comportamento da força ao longo do tempo (Figura 5A, B). Porém não se verificou diferença na AUC o que conclui que a variação das frequências utilizadas não é fator principal no processo de fadiga muscular. Com isso se verificou uma possível ação causal das diferentes frequências na EENM.

A eletromiografia de superfície (EMG) pode ser utilizada durante protocolos de indução à fadiga para estimar um padrão de atividade muscular baseado nas informações fisiológicas que ela fornece. Com base nisto, um fenômeno bastante observado é a diminuição da velocidade de condução dos potenciais de ação nas fibras musculares durante uma contração muscular. A Fmd pode ser um parâmetro utilizado para identificar alteração no sinal eletromiográfico, que permite avaliar o comportamento dos músculos ao longo do método de indução à fadiga.

Durante esse processo, além das alterações na modulação da frequência de disparo também ocorrem alterações na sincronização e recrutamento de unidades motoras. Para manter o nível de contração desejado, durante um teste de indução à fadiga, ocorre um aumento de recrutamento de unidades motoras, aumentando a amplitude do sinal eletromiográfico. O que conclui que a queda do sinal RMS e a queda da Fmd resultam em Fadiga. Foi avaliado o comportamento EMG durante a CVM após EENM, através do RMS e Fmd que corresponderiam os efeitos do stress muscular após a EENM. Complementando aos resultados dos dados EMG, os valores de RMS e Fmd obtiveram resultados iguais em todas as frequências, o que significa que não foram capazes de gerar fadiga, fazendo com que a utilização das frequências seja segura. (JEFFREY R. CRAM; GLENN S. KASMAN, 1998)

No atual estudo, as Tabelas 2 e 3, que utilizaram RMS e Fmd e correspondem ao estímulo gerado pela EENM, não foram capazes de detectar a instalação de fadiga nas frequências utilizadas. As frequências não são fatores principais para instalação precoce da fadiga.

O exercício físico induz a uma maior necessidade de energia, logo a Glicose é uma fonte para obtenção de energia. A contração muscular induz a ativação das IRS. Após a auto fosforilação dessas proteínas é liberada uma cascata de reações, liberando o AKT que é o ativador da proteína transportadora de Glicose, o GLUT-4. O GLUT-4 se transloca para a membrana celular, fazendo o transporte da Glicose sanguínea para o meio intracelular, fazendo com que os níveis de glicose sanguíneo diminuam (ROPELLE ER, PAULI JR, 2005). Com base nesse processo de transporte da Glicose e queda dos níveis da glicose, foi observado que os resultados das frequências $60 \mathrm{~Hz}$ e $120 \mathrm{~Hz}$ na Corrente AUSSIE com a capacidade Hipoglicemiante $(\mathrm{p}<0,05)$. Foram realizados os Intervalos de Confiança (Sidak) correspondente a 95\%, que indica a diferença clínica através da análise glicêmica realizada no estudo, onde a maior diferença entre as médias se encontra em $60 \mathrm{~Hz}$ e $120 \mathrm{~Hz}$, gerando maior confiabilidade que essas frequências tem impacto na prática clínica. A frequência $90 \mathrm{~Hz}$ da Corrente AUSSIE não obteve diferença, apesar de quase se igualar ao efeito hipoglicemiante de $60 \mathrm{~Hz}$ e $120 \mathrm{~Hz}$. A possível hipótese desse evento, é pelo baixo número de indivíduos no atual estudo, necessitando de uma nova pesquisa com um número maior de indivíduos para verificar se esse comportamento se manterá.

$\mathrm{Na}$ corrente alternada as frequências $90 \mathrm{hz}$ e $120 \mathrm{~Hz}$ também demonstram capacidade Hipoglicemiante $(\mathrm{p}<0,05)$ (Figura $6 \mathrm{~B})$, onde foram feitos os Intervalos de Confiança (Bonferroni)

Persp. online: biol. \& saúde, Campos dos Goytacazes, 28 (8), 1-14, 2018 
com $95 \%$, onde a maior diferença entre as médias se encontra na frequência de $120 \mathrm{~Hz}$, onde garante que a aplicabilidade da frequência tem impacto na prática clínica.

A possível explicação para esse evento se encontra em um estudo que investigou as vias de sinalização do músculo esquelético quando associadas à translocação do GLUT-4, tanto em repouso, quanto após treinamento de resistência induzido por EENM divididos em dois grupos, os indivíduos com lesão medular e os indivíduos com total função músculo esquelética. A conclusão do estudo foi que a fosforilação de proteínas de sinalização (Adenosina monofosfato ativada por proteína quinase (AMPK)) são positivamente reguladas em relação a translocação do GLUT-4, mesmo em indivíduos lesionados medulares. A sinalização pode ser uma tentativa compensatória aos baixos níveis de concentração de GLUT-4 no músculo (YARAR-FISHER et al., 2013b). O que mostra a principal participação da contração muscular para a realização desse evento.

\section{CONCLUSÃO}

Com a metodologia proposta pode-se averiguar que as diferentes frequências da EENM não foram capazes de promover alterações na produção de força isométrica induzida eletricamente e nem na geração de fadiga muscular pós EENM. Porém verificou-se que as frequências exercem papel importante na geração de estresse metabólico proporcionando maior consumo de glicose.

Sugere-se estudos futuros com pacientes diabéticos com objetivo de melhor explicar esses achados e avaliar o seu potencial de aplicação terapêutico. Sugere-se também a aplicação da mesma metodologia com maior número de participantes a fim de se consolidar esse conhecimento.

\section{REFERÊNCIAS}

AGNE, J. E. Eletrotermoterapia: teoria e prática. $1^{\circ}$ ed. [s.1.] Orium Editora \& Comunicação Ltda., 2004.

AGNE, J. E. Eletrotermofototerapia. $2^{\circ}$ ed. Santa Maria: O AUTOR, 2015.

DIRKS, M. L. et al. Neuromuscular electrical stimulation prevents muscle disuse atrophy during leg immobilization in humans. Acta Physiologica, 2014.

ENGLISH, K. L.; PADDON-JONES, D. Protecting muscle mass and function in older adults during bed rest. [s.d.].

FINSTERER, J.; MAHJOUB, S. Z. Fatigue in Healthy and Diseased Individuals. American Journal of Hospice and Palliative Medicine, v. 31, n. 5, p. 562-575, 1 ago. 2014.

HAYASHI, T.; WOJTASZEWSKI, J. F.; GOODYEAR, L. J. Exercise regulation of glucose transport in skeletal muscle. The American journal of physiology, v. 273, n. 6 Pt 1, p. E1039-51, dez. 1997.

JEFFREY R. CRAM; GLENN S. KASMAN. Introduction to Surface Electromyography. $1^{\text {a }}$ ed. USA: Aspen Publishers, 1998.

Persp. online: biol. \& saúde, Campos dos Goytacazes, 28 (8), 1-14, 2018

seer.perspectivasonline.com.br 
LANGEARD, A. et al. Does neuromuscular electrical stimulation training of the lower limb have functional effects on the elderly?: A systematic review. Experimental Gerontology, v. 91, p. 88-98, 2017.

LIEBANO, R. E.; WASZCZUK, S.; CORRÊA, J. B. The Effect of Burst-Duty-Cycle Parameters of Medium-Frequency Alternating Current on Maximum Electrically Induced Torque of the Quadriceps Femoris, Discomfort, and Tolerated Current Amplitude in Professional Soccer Players. Journal of Orthopaedic \& Sports Physical Therapy, v. 43, n. 12, p. 920-926, 2013.

LUCIANO, E. et al. Endurance training improves responsiveness to insulin and modulates insulin signal transduction through the phosphatidylinositol 3-kinase/Akt-1 pathway. European Journal of Endocrinology, v. 147, n. 1, p. 149-157, 2002.

MCARLE, W. D.; KATCH, F. I.; KATCH, V. L. Fisiologia do exercício: Energia, Nutrição e Desempenho humano. 5. ed. Rio de Janeiro: Guanabara Koogan S.A., 2003.

MITCHELL, R. N. ET AL. Fundamentos de Robbins \& Cotran: patologia. $8^{\circ}$ ed. Rio de Janeiro: Elsevier, 2012.

POWERS, S. K.; HOWLEY, E. T. Fisiologia do exercício: Teoria e aplicação ao condicionamento e ao desempenho. 6. ed. São Paulo: Manole, 2009.

PRATT, C.; VOET, D.; VOET, J. G. Fundamentos de Bioquímica: a vida em nível molecular. 2. ed. Porto Alegre: Artimed, 2008.

ROPELLE ER, PAULI JR, C. J. Efeitos moleculares do exercício físico sobre as vias de sinalização insulínica Exercício e sinalização insulínica : perspectiva histórica Efeitos do exercício físico na via de sinalização da insulina. Motriz, v. 11, n. 1, p. 49-55, 2005.

THEILEN, N. T.; KUNKEL, G. H.; TYAGI, S. C. The Role of Exercise and TFAM in Preventing Skeletal Muscle Atrophy. Journal of Cellular Physiology, v. 232, n. 9, p. 2348-2358, 2017.

WALL, B. T. et al. Neuromuscular electrical stimulation increases muscle protein synthesis in elderly type 2 diabetic men. American Journal of physiology - Endocrinology and Metabolism, v. 303, n. 36, p. E614-23, 2012.

WARD, A. R.; OliVER, W. G.; BUCCELlA, D. Wrist Extensor Torque Production and Discomfort Associated With Low-Frequency and Burst-Modulated Kilohertz-Frequency Currents. Physical Therapy, v. 86, n. 10, p. 1360-1367, 2006.

WARD, A. R.; ROBERTSON, V. J.; IOANNOU, H. The effect of duty cycle and frequency on muscle torque production using kilohertz frequency range alternating current. Medical Engineering and Physics, v. 26, n. 7, p. 569-579, 2004.

YARAR-FISHER, C. et al. Skeletal muscle signaling associated with impaired glucose tolerance in spinal cord-injured men and the effects of contractile activity. Journal of applied physiology (Bethesda, Md. : 1985), v. 115, n. 5, p. 756-64, $2013 \mathrm{a}$. 